\title{
SUBARU HIGH-RESOLUTION SPECTROSCOPY OF COMPLEX METAL ABSORPTION LINES OF THE QUASAR HS $1603+3820^{1}$
}

\author{
Toru Misawa, ${ }^{2}$ Toru Yamada,${ }^{3}$ Masahide Takada-Hidai, ${ }^{4}$ Yiping Wang, ${ }^{5}$ Nobunari KashiKawa, ${ }^{3}$ \\ MASANORI IYE, ${ }^{3,6}$ AND ICHI TANAKA ${ }^{3}$ \\ Received 2002 September 26; accepted 2002 November 19
}

\begin{abstract}
We present a high-resolution spectrum of the quasar HS $1603+3820\left(z_{\mathrm{em}}=2.542\right)$, observed with the High Dispersion Spectrograph on the Subaru Telescope. This quasar, first discovered in the Hamburg/CfA Quasar Survey, has $11 \mathrm{C}$ IV lines at $1.96<z_{\text {abs }}<2.55$. Our spectrum covers eight of the $11 \mathrm{C}$ iv lines at $z_{\text {abs }}>2.29$ and resolves some of them into multiple narrow components with $b<25 \mathrm{~km} \mathrm{~s}^{-1}$ because of the high spectral resolution $R=45,000$, while other lines show broad profiles $\left(b>65 \mathrm{~km} \mathrm{~s}^{-1}\right)$. We use three properties of $\mathrm{C}$ IV lines, specifically, time variability, covering factor, and absorption-line profile, to classify them into quasar intrinsic absorption lines (QIALs) and spatially intervening absorption lines (SIALs). The $\mathrm{C}$ IV lines at $2.42<z_{\mathrm{abs}}<2.45$ are classified as QIALs in spite of their large velocity shifts from the quasar. Perhaps they are produced by gas clouds ejected from the quasar with velocity $v_{\mathrm{ej}}=8000-10,000 \mathrm{~km} \mathrm{~s}^{-1}$. On the other hand, three $\mathrm{C}$ IV lines at $2.48<z_{\text {abs }}<2.55$ are classified as SIALs, which suggests there exist intervening absorbers near the quasar. We, however, cannot rule out QIALs for the two lines at $z_{\mathrm{abs}} \sim 2.54$ and 2.55 , because their velocity shifts, $430 \mathrm{~km} \mathrm{~s}^{-1}$ blueward and $950 \mathrm{~km} \mathrm{~s}^{-1}$ redward of the quasar, are very small. The $\mathrm{C}$ iv line at $z_{\mathrm{abs}} \sim 2.48$ consists of many narrow components and also has corresponding low-ionization metal lines ( $\mathrm{Al}$ II, Si II, and Fe II). The velocity distribution of these low-ionization ions is concentrated at the center of the system compared with that of the high-ionization $\mathrm{C}$ IV ion. Therefore we ascribe this system of absorption lines to an intervening galaxy.
\end{abstract}

Key words: quasars: absorption lines — quasars: individual (HS 1603+3820)

\section{INTRODUCTION}

The bright quasar HS $1603+3820 \quad\left(z_{\mathrm{em}}=2.542\right.$, $B=15.9$ ), first discovered in the Hamburg/CfA Bright Quasar Survey (Hagen et al. 1995; Dobrzycki et al. 1996), has very unusual properties, namely, the combination of high redshift, large luminosity, and richness of metal absorption lines. In the spectrum of this quasar, $11 \mathrm{C}$ IV absorption lines were detected at $1.965<z_{\text {abs }}<$ 2.554 (Dobrzycki, Engels, \& Hagen 1999, hereafter D99). Among the $11 \mathrm{C}$ IV lines, eight lines are observed at $2.420<z_{\text {abs }}<2.554$, which corresponds to a velocity shift of $-10,600 \mathrm{~km} \mathrm{~s}^{-1}<\Delta v<+1000 \mathrm{~km} \mathrm{~s}^{-1}$ from the quasar. The number density of $\mathrm{C}$ IV lines with restframe equivalent width $W_{\mathrm{rf}} \geq 0.15$ is $N(z) \sim 21$ per unit redshift at $z_{\mathrm{abs}} \sim 2.38$ with $\Delta z=0.34$. If we combine $\mathrm{C}$ Iv lines that lie within $1000 \mathrm{~km} \mathrm{~s}^{-1}$ of each other to produce a so-called Poisson sample (see Sargent, Boksenberg, \& Steidel 1988), $N(z)$ becomes $\sim 12$, which is significantly larger than the expected average value,

${ }^{1}$ Based on data collected at the Subaru Telescope, which is operated by the National Astronomical Observatory of Japan.

${ }^{2}$ Department of Astronomy, School of Science, University of Tokyo, 7-3-1 Hongo, Bunkyo-ku, Tokyo 113-0033, Japan; misawatr@cc.nao.ac.jp.

${ }^{3}$ National Astronomical Observatory, 2-21-1 Osawa, Mitaka, Tokyo 181-8588, Japan.

${ }^{4}$ Liberal Arts Education Center, Tokai University, 1117 Kitakaname, Hiratsuka, Kanagawa 259-1292, Japan.

${ }_{5}^{5}$ Purple Mountain Observatory, National Astronomical Observatories, Chinese Academy of Sciences, 2 Beijing Xi Lu, Nanjing, Jiangsu 210008, China.

${ }^{6}$ Department of Astronomical Science, Graduate University for Advanced Studies, 2-21-1 Osawa, Mitaka, Tokyo 181-8588, Japan.
$N(z)=2.45_{-0.49}^{+0.60}$, at $z_{\text {abs }} \sim 2.40$ evaluated for $\mathrm{C}$ IV lines with $W_{\text {rf }} \geq 0.15 \AA$ and $v_{\text {ej }}>5000 \mathrm{~km} \mathrm{~s}^{-1}$ in previous studies (e.g., Sargent et al. 1988; Steidel 1990; Misawa et al. 2002). Such an extreme overdensity of C IV lines inspired us to obtain a high-resolution spectrum of this quasar with the Subaru Telescope for a detailed study of the $\mathrm{C}$ IV lines.

Absorbers are generally divided into quasar intrinsic absorption lines (QIALs) and spatially intervening absorption lines (SIALs). QIALs are thought to be produced by gas clouds intrinsically associated with the nuclear regions of the quasars. On the other hand, SIALs are produced by either intervening galaxies located along the line of sight of the quasars or the interstellar matter in the quasar host galaxies. It is essential to understand the origin of the absorption lines to answer questions such as what causes the extremely high number density of $\mathrm{C}$ IV lines in the spectrum of HS $1603+3820$.

To distinguish QIALs from SIALs, we use the seven proper criteria (Barlow \& Sargent 1997; Hamann et al. 1997b; references therein) that have been proposed in the literature:

1. Time variability of equivalent width is sometimes detected in QIALs.

2. QIALs are produced by absorbers with high electron space density.

3. Absorbers that produce QIALs partially cover the background of the quasar.

4. The polarization rate in QIALs is larger than that in the continuum.

5. QIALs have smoother and broader line profiles than those of SIALs. 
6. QIALs are produced by highly ionized absorbers.

7. QIALs are produced by absorbers with high metallicity.

With one or a combination of these criteria, for example, Barlow et al. (1992) found that the broad absorption line (BAL) with FWHM $\geq 100 \mathrm{~km} \mathrm{~s}^{-1}$ near the emission redshift of CSO 203 is a QIAL, because the line shows time variability. Hamann, Barlow, \& Junkkarinen (1997a) also found that the broad C IV doublet even at $-24,000 \mathrm{~km} \mathrm{~s}^{-1}$ from Q2343+125 shows time variability. Barlow \& Sargent (1997) and Hamann et al. (1997b) found QIALs by the covering factor analysis in PKS $0123+257$ and UM 675. Goodrich \& Miller (1995) used the polarization method to distinguish QIALs from SIALs in PHL 5200 and $\mathrm{H} 1413+117$.

For HS $1603+3820$, D99 used only the covering factor analysis with their low-resolution spectrum. They applied the method to the broad C Iv line at the high ejection velocity end of the C IV complex and found that it is a QIAL. However, the other lines were not classified, because they have line profiles narrower than the spectral resolution or maybe they are affected by other lines.

In this paper, we present a high-resolution $(R \sim 45,000)$ spectrum of HS $1603+3820$ obtained with the High Dispersion Spectrograph (HDS; Noguchi et al. 2002) on the Subaru Telescope. The C Iv lines are found to have further complex structure, and we classify them into QIALs and SIALs to discuss the cause of their large number density. In $\S 2$, we describe the observations and data reduction. In $\S 3$, the properties of C IV systems are examined, and the classification of C IV systems is delineated in $\S 4$. Results and discussion are given in $\S 5$. We present the conclusions and the prospect of the future work in $\S 6$.

\section{OBSERVATION AND DATA REDUCTION}

We carried out spectroscopic observation of the quasar HS $1603+3820\left(\alpha=16^{\mathrm{h}} 04^{\mathrm{m}} 55^{\prime} 4, \delta=+38^{\circ} 12^{\prime} 01^{\prime \prime}[\mathrm{J} 2000.0]\right)$ with HDS on the Subaru Telescope on 2002 March 23 (UT).

It has to be noted that the Ly $\alpha$ emission line is affected by complex metal absorption lines, which makes the value of emission-line redshift of the quasar uncertain (D99). D99 suggested the emission redshift as $z_{\mathrm{em}} \sim 2.51$ by estimating a continuum around the Ly $\alpha$ and $\mathrm{C}$ IV emission lines and considering the fact that the redshifts determined from the broad emission lines can be blueshifted by $\geq 1200 \mathrm{~km} \mathrm{~s}^{-1}$ with respect to the systemic redshift from the narrow forbidden lines (Espey 1993). If we match the spectrum of the quasar taken by D99 to the SDSS composite spectrum (Vanden Berk et al. 2001) by using O I $\lambda 1304$ and Si II $\lambda 1307$ and $\mathrm{C}_{\text {II }}$ $\lambda 1335$ emission lines, the systemic redshift of the quasar is estimated to be $z_{\mathrm{em}}=2.542 \pm 0.003$. Richards et al. (2002b) also showed that quasars with weaker $\mathrm{C}$ IV emission lines tend to be greatly blueshifted, in excess of $2000 \mathrm{~km} \mathrm{~s}^{-1}$. Since the C IV emission line of HS $1603+3820$ is very weak, the systemic redshift of the quasar may be larger than $z_{\mathrm{em}}=2.51$. Therefore we assume $z_{\mathrm{em}}$ of the quasar to be 2.542 instead of $z_{\mathrm{em}}=2.51$ throughout this paper.

HDS has two $2 \mathrm{~K} \times 4 \mathrm{~K} C \mathrm{CDs}$, which are the blue and red CCDs. We adopted 2 pixel binning along the slit, and we used the red grating with a central wavelength of $6450 \AA$, which covers 5100-6400 $\AA$ on the blue CCD and 6500-7600
$\AA$ on the red CCD. The results discussed in this paper are based on the spectra taken by the blue CCD alone. The slit width of 0 " 8 provides the spectral resolution of $45,000(6.67$ $\mathrm{km} \mathrm{s}^{-1}$ ). The weather conditions were very good, and the seeing was $\sim 0$ ". 5 . The signal-to-noise ratio of the spectrum with 2700 s exposure was $\sim 40$ per pixel around $\lambda=5450 \AA$.

The data were reduced with the IRAF package in the standard manner. We determined the continuum by fitting the data with a third-order cubic spline function. The spectrum has a heavily absorbed region around $5300-5350 \AA$ in the 20th echelle order (Fig. 1), and it is not straightforward to determine the continuum level there. In such case, we evaluated the continuum profile $q_{i}(m)$ of the heavily absorbed order $i$ by

$$
q_{i}(m)=\frac{1}{2}\left[\frac{f_{i}(m)}{f_{i-1}(m)} q_{i-1}(m)+\frac{f_{i}(m)}{f_{i+1}(m)} q_{i+1}(m)\right],
$$

where $f_{i-1}(m), f_{i}(m)$, and $f_{i+1}(m)$ are the counts at the $m$ th pixel of order $i-1, i$, and $i+1$, respectively, of the continuum profile fitted for the flat-frame spectrum and $q_{i-1}(m)$ and $q_{i+1}(m)$ are the counts of order $i-1$ and $i+1$ of the continuum profile fitted for the quasar spectrum, respectively. We acquired the normalized $i$ th order spectrum by dividing the quasar spectrum of order $i$ by $q_{i}(m)$. We used order 17 and 23 to produce $q_{20}(m)$, because orders 18,19 , 21 , and 22 are weakly affected by absorption lines. In Figure

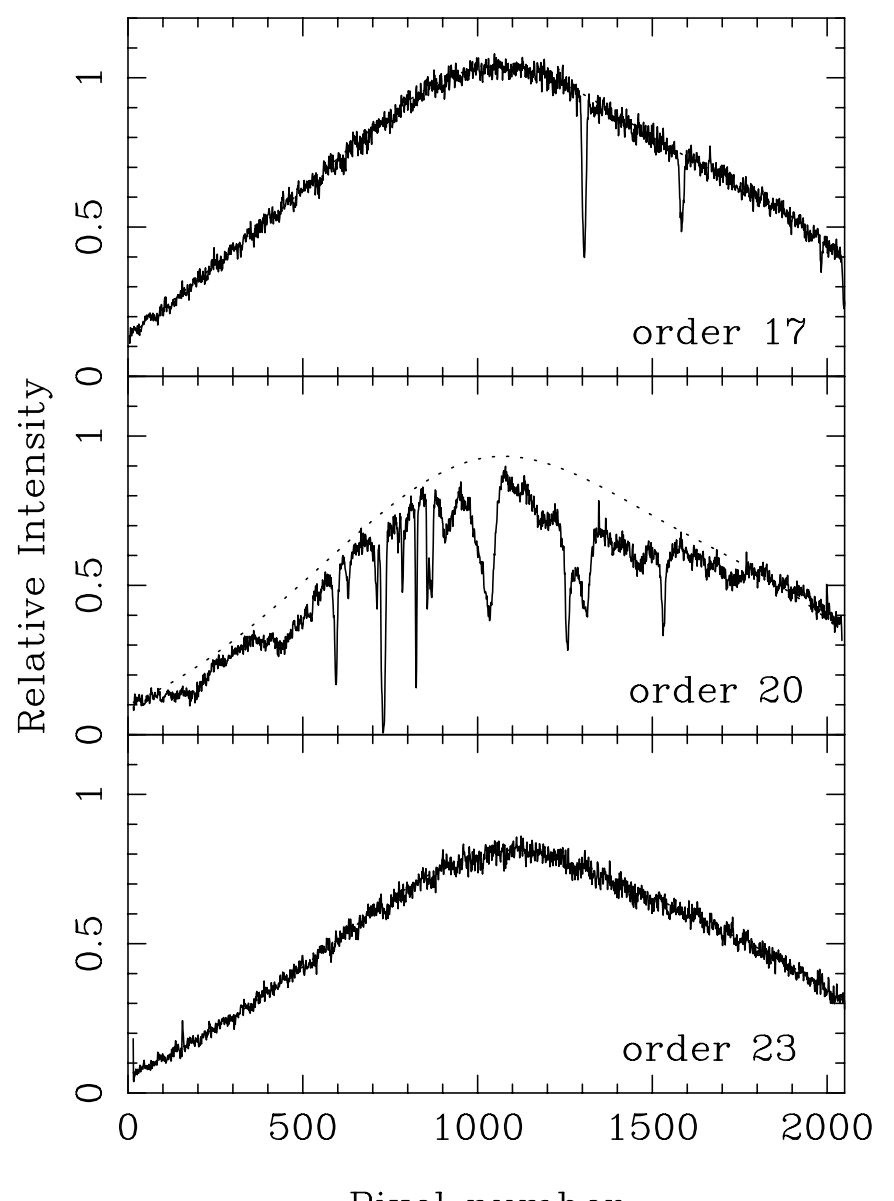

Pixel number

FIG. 1.- Spectrum of HS $1603+3820$ at echelle orders of 17, 20, and 23 before normalization. With order 17 and 23 , the continuum function of order 20 is interpolated (middle, dotted line). 

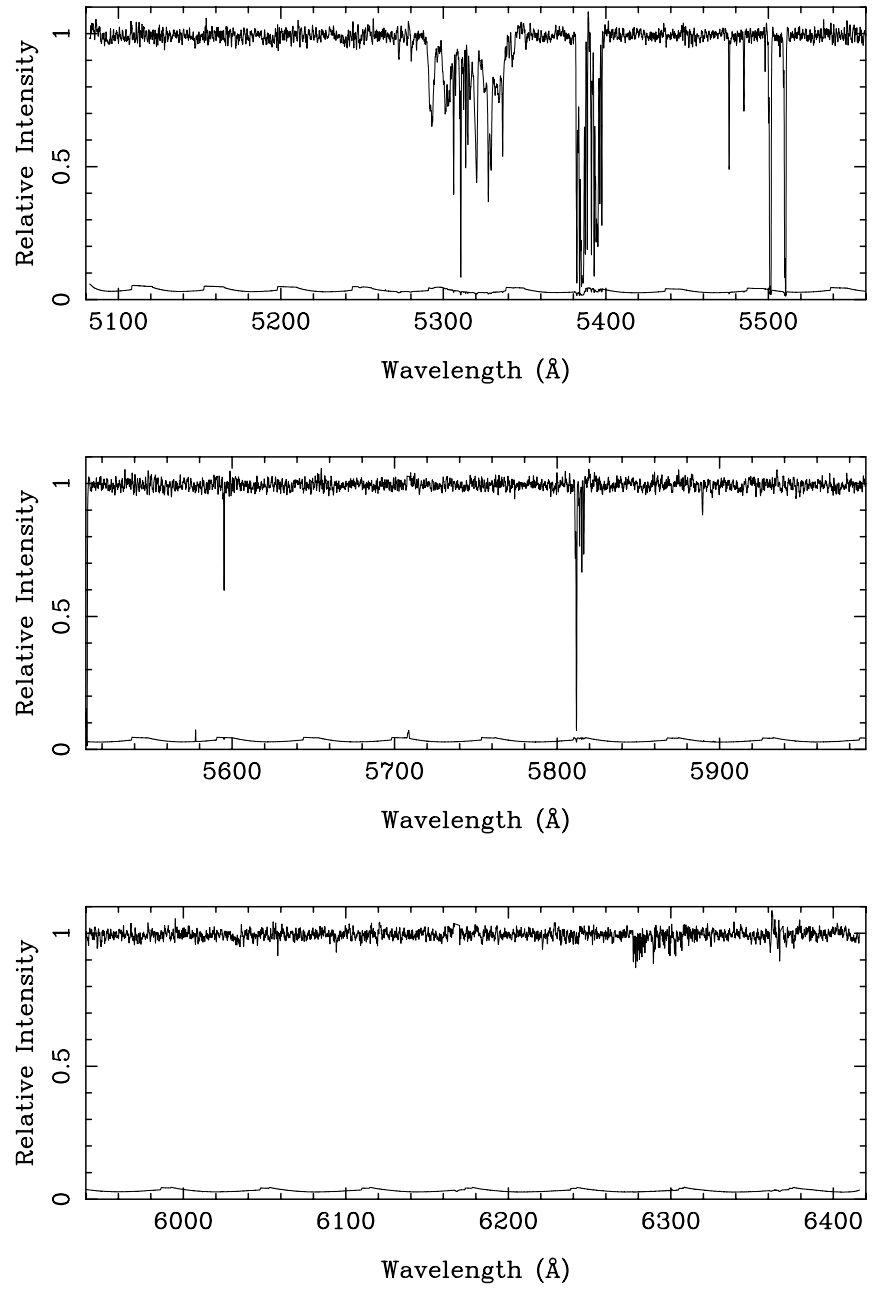

FIG. 2.-Normalized Spectrum of HS 1603 smoothed by 3 pixels. The obvious cosmic rays are chopped. The lower spectrum in each panel is the $1 \sigma$ error level.

1 (middle) the interpolated continuum of order $20, q_{20}(\mathrm{~m})$, is shown as a dotted line. For confirmation of the validity of this technique, we applied this method to the stellar spectrum used to locate the positions and track the shapes of all the echelle orders. The continuum flux of order $i$ obtained with this interpolation method is only 3.3\% smaller on average than that of the directly fitted spectrum. Therefore, we believe this technique is sufficiently useful for our study. The normalized spectrum of each order is combined to produce the final spectrum (Fig. 2).

\section{IDENTIFICATION OF C IV SYSTEMS}

We used the VPFIT Voigt profile line-fitting software developed by Carswell et al. (Webb 1987; Carswell et al. 1987). D99 detected $11 \mathrm{C}$ iv lines at $1.96<z_{\mathrm{abs}}<2.55$. Our spectrum covers eight of them in the range $2.42<$ $z_{\text {abs }}<2.55$ and detected absorption lines at the wavelength of all these lines. Thanks to high spectral resolution, some $\mathrm{C}$ Iv lines detected in D99 are further resolved into multiple narrow components, resulting in a total of $30 \mathrm{C}$ IV components between $z=2.42$ and 2.55. Figure 3 shows both the HDS and MMT (Multiple Mirror Telescope) spectra

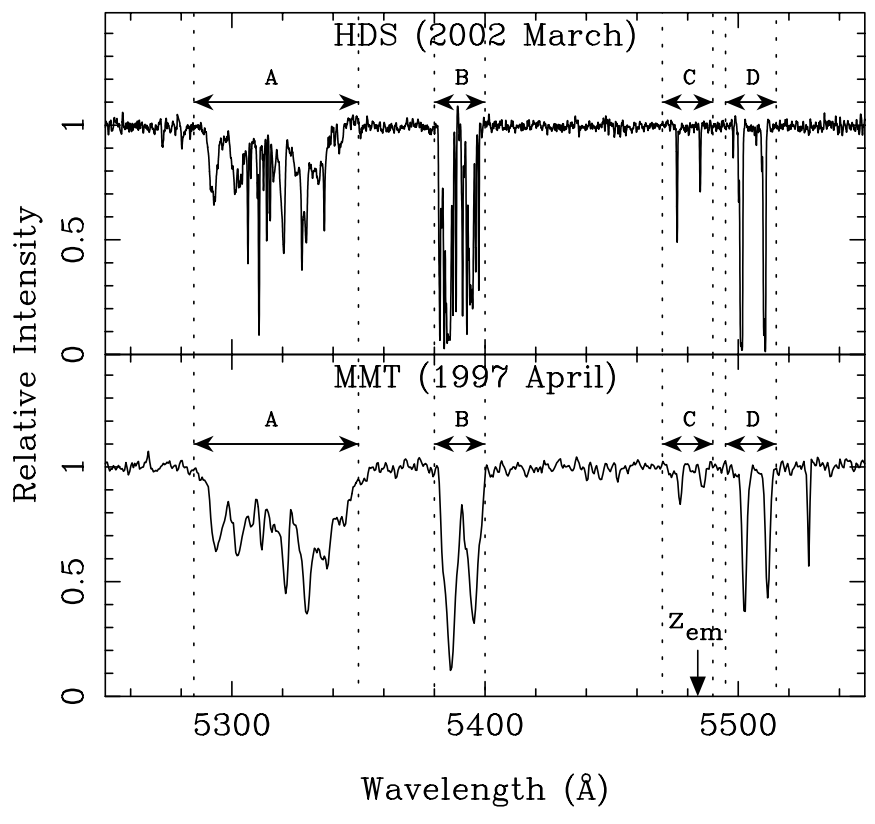

FIG. 3.-HDS spectrum with $R=45,000$, compared with MMT spectrum with $R \sim 3000$ (D99). The four C IV systems are surrounded by dotted lines at each side. The Fe II 1608 line around $5525 \mathrm{~A}$ in the MMT spectrum disappears in the HDS spectrum.

(D99). ${ }^{7}$ We summarize the results in Table 1. Column (1) lists the metal line identification and ID number. Columns (2) and (3) list the observed wavelength and redshift. Column (4) lists the velocity difference from $z_{\mathrm{em}}$, and column (5) lists the Doppler parameter, $b=\sqrt{2} \sigma$ (where $\sigma$ is the velocity dispersion). Columns (6)-(9) list the covering factor, optical depth at line center, and column densities for covering factors $C_{f}=1$ and $C_{f}<1$, which are discussed in $\S 4$ in detail.

For the sake of simplicity, we divided these $\mathrm{C}$ iv lines into four groups, system A to system D as shown in Figure 3. The C Iv lines in systems B and D in D99 are resolved into multiple narrow components with $b<25 \mathrm{~km} \mathrm{~s}^{-1}$ in our spectrum. The line in system $\mathrm{C}$ is not resolved, but this line has a small Doppler value $b=11 \mathrm{~km} \mathrm{~s}^{-1}$. On the other hand, the five C IV lines clustering in system A in D99 are not further resolved into narrow components, although three of them have broad-line profiles, $b>70 \mathrm{~km} \mathrm{~s}^{-1}$. Detailed notes on each system are described below.

System $A\left(2.42<z_{\mathrm{abs}}<2.45\right)$. D99 detected five C IV lines in this system at velocity shift $-10,600 \mathrm{~km}$ $\mathrm{s}^{-1}<\Delta v<-7800 \mathrm{~km} \mathrm{~s}^{-1}$ from $z_{\mathrm{em}}$ in their spectrum. We found two additional $\mathrm{C}$ Iv lines in our spectrum, as shown in Figure 4 . In addition to these lines, there still remains the broad absorption feature at $-9300 \mathrm{~km} \mathrm{~s}^{-1}<\Delta v<-8000$ $\mathrm{km} \mathrm{s}^{-1}$. We cannot separate the lines to fit the Voigt profile individually because they are heavily blended with each other. Five of the seven detected C IV lines $\left(z_{\mathrm{abs}}=2.4186\right.$, $2.4257,2.4341,2.4362$, and 2.4510 ) are relatively broad and smooth, and their appearances are quite similar. On the other hand, the line $\left(z_{\mathrm{abs}}=2.4366\right)$ has a relatively narrowline profile. Perhaps it is an independent system of the broad $\mathrm{C}$ IV lines. Another interesting result is that $\mathrm{Fe}$ II $\lambda 1608$ at

${ }^{7}$ See http://hea-www.harvard.edu/QEDT/Papers/hs 1603 
TABLE 1

List of Metal Absorption Lines

\begin{tabular}{|c|c|c|c|c|c|c|c|c|}
\hline $\begin{array}{l}\text { Line ID } \\
\text { (1) }\end{array}$ & $\begin{array}{l}\lambda_{\text {obs }} \\
(\AA) \\
(2)\end{array}$ & $\begin{array}{l}z_{\text {abs }} \\
(3)\end{array}$ & $\begin{array}{c}V \\
(4)\end{array}$ & $\begin{array}{c}b \\
\left(\mathrm{~km} \mathrm{~s}^{-1}\right) \\
(5)\end{array}$ & $\begin{array}{l}C_{f} \\
(6)\end{array}$ & $\begin{array}{l}\tau^{\mathrm{a}} \\
(7)\end{array}$ & $\begin{array}{c}\log N \text { for } C_{f}=1 \\
\left(\mathrm{~cm}^{-2}\right) \\
(8)\end{array}$ & $\begin{array}{c}\log N \text { for } C_{f}<1 \\
\left(\mathrm{~cm}^{-2}\right) \\
\text { (9) }\end{array}$ \\
\hline \multicolumn{9}{|c|}{ System A, $z_{\text {abs }}=2.44$} \\
\hline C IV $\lambda 1548:$ & & & & & & & & \\
\hline $1 \ldots \ldots \ldots \ldots$ & 5292.7 & 2.4186 & -10634 & $114.28 \pm 1.96$ & $0.31 \pm 0.03^{b}$ & $4.32_{-2.03}^{\infty} \mathrm{b}$ & 14.23 & $14.80_{-0.28}^{\infty} \mathrm{b}$ \\
\hline $2 \ldots \ldots \ldots \ldots$ & 5303.8 & 2.4257 & -10008 & $77.68 \pm 5.59^{\mathrm{c}}$ & $\ldots$ & $\ldots$ & $\ldots$ & $\ldots$ \\
\hline $3 \ldots \ldots \ldots \ldots$ & 5316.7 & 2.4341 & -9276 & $65.19 \pm 2.85^{\mathrm{c}}$ & $\ldots$ & $\ldots$ & $\cdots$ & $\cdots$ \\
\hline $4 \ldots \ldots \ldots \ldots$ & 5320.0 & 2.4362 & -9092 & $80.06 \pm 1.52^{\mathrm{c}}$ & $\ldots$ & $\ldots$ & $\ldots$ & $\ldots$ \\
\hline $5 \ldots \ldots \ldots \ldots$ & 5320.6 & 2.4366 & -9058 & $25.30 \pm 1.16^{\mathrm{c}}$ & & & & \\
\hline $6 \ldots \ldots \ldots \ldots$ & 5327.7 & 2.4412 & -8656 & $15.68 \pm 0.31$ & 0.796 & 1.912 & 13.4 & 13.6 \\
\hline $7 \ldots \ldots \ldots \ldots$ & 5342.9 & 2.4510 & -7806 & $74.36 \pm 6.06^{\mathrm{c}}$ & $\ldots$ & $\ldots$ & $\ldots$ & $\ldots$ \\
\hline
\end{tabular}

System B, $z_{\text {abs }}=2.48$

\begin{tabular}{|c|c|c|c|c|c|c|c|c|}
\hline \multicolumn{9}{|l|}{ C IV $\lambda 1548:$} \\
\hline $1 \ldots \ldots \ldots \ldots$ & 5381.8 & 2.4762 & -5628 & $9.62 \pm 0.27$ & 1.000 & 1.169 & 13.2 & $\ldots$ \\
\hline $2^{\mathrm{d}} \ldots \ldots \ldots$ & 5382.2 & 2.4764 & -5608 & $7.39 \pm 0.11$ & 1.000 & & $14.0^{\mathrm{e}}$ & $\ldots$ \\
\hline $3 \ldots \ldots \ldots \ldots$ & 5382.8 & 2.4768 & -5573 & $20.42 \pm 1.92$ & 1.000 & 0.607 & 13.2 & $\ldots$ \\
\hline $4 \ldots \ldots \ldots \ldots$ & 5383.0 & 2.4769 & -5560 & $4.62 \pm 0.60$ & 1.000 & 0.702 & 12.6 & $\ldots$ \\
\hline $5 \ldots \ldots \ldots \ldots$ & 5383.3 & 2.4771 & -5544 & $7.57 \pm 2.09$ & 1.000 & 0.328 & 12.5 & \\
\hline $6 \ldots \ldots \ldots \ldots$ & 5383.8 & 2.4774 & -5519 & $13.49 \pm 4.94$ & 0.977 & 5.653 & 13.8 & 14.0 \\
\hline $7^{\mathrm{b}} \ldots \ldots \ldots$ & 5383.9 & 2.4775 & -5512 & $7.76 \pm 0.54$ & 1.000 & & $14.1^{\mathrm{e}}$ & \\
\hline $8 \ldots \ldots \ldots \ldots$ & 5384.2 & 2.4777 & -5494 & $8.84 \pm 2.62$ & 0.783 & 0.958 & 12.9 & 13.1 \\
\hline $9 \ldots \ldots \ldots \ldots$ & 5384.5 & 2.4779 & -5477 & $9.63 \pm 0.53$ & 0.985 & 2.416 & 13.5 & 13.5 \\
\hline $10 \ldots \ldots \ldots$ & 5385.0 & 2.4782 & -5450 & $12.37 \pm 0.44$ & 1.000 & 3.258 & 13.7 & \\
\hline $11 \ldots \ldots \ldots$ & 5385.6 & 2.4786 & -5416 & $24.03 \pm 2.29$ & 0.997 & 3.128 & 14.0 & 14.0 \\
\hline $12 \ldots \ldots \ldots \ldots$ & 5386.2 & 2.4790 & -5385 & $10.65 \pm 1.25$ & 0.980 & 4.072 & 13.7 & 13.8 \\
\hline $13 \ldots \ldots \ldots$ & 5386.4 & 2.4791 & -5373 & $20.78 \pm 3.19$ & 0.985 & 1.917 & 13.7 & 13.7 \\
\hline $14 \ldots \ldots \ldots \ldots$ & 5387.1 & 2.4795 & -5336 & $8.48 \pm 0.81$ & 1.000 & 0.645 & 12.9 & $\ldots$ \\
\hline $15 \ldots \ldots \ldots$ & 5387.4 & 2.4797 & -5319 & $8.57 \pm 0.24$ & 1.000 & 2.870 & 13.5 & \\
\hline $16 \ldots \ldots \ldots \ldots$ & 5388.3 & 2.4803 & -5268 & $23.13 \pm 1.43$ & $0.277^{\mathrm{f}}$ & 0.901 & 12.8 & 13.5 \\
\hline $17 \ldots \ldots \ldots$ & 5388.6 & 2.4805 & -5251 & $5.47 \pm 0.12$ & 0.987 & 5.931 & 13.5 & 13.6 \\
\hline $18 \ldots \ldots \ldots \ldots$ & 5389.8 & 2.4813 & -5182 & $14.49 \pm 2.02$ & 1.000 & 0.131 & 12.4 & $\ldots$ \\
\hline \multicolumn{9}{|l|}{$\mathrm{Al}$ II $\lambda 1670:$} \\
\hline $1 \ldots \ldots \ldots \ldots$ & 5811.2 & 2.4781 & -5458 & $12.09 \pm 0.65$ & $>0.336$ & $>0.410$ & 11.8 & $\ldots$ \\
\hline $2^{\mathrm{g}} \ldots \ldots \ldots \ldots$ & 5811.9 & 2.4785 & -5422 & $9.69 \pm 0.16$ & 1.000 & & $12.9^{\mathrm{e}}$ & $\cdots$ \\
\hline $3 \ldots \ldots \ldots \ldots$ & 5813.4 & 2.4794 & -5345 & $5.25 \pm 1.13$ & $>0.140$ & $>0.151$ & 11.0 & $\ldots$ \\
\hline $4 \ldots \ldots \ldots \ldots$ & 5813.9 & 2.4797 & -5322 & $6.37 \pm 0.44$ & $>0.361$ & $>0.448$ & 11.6 & $\ldots$ \\
\hline $5 \ldots \ldots \ldots \ldots$ & 5815.2 & 2.4805 & -5251 & $4.00 \pm 0.19$ & $>0.583$ & $>0.875$ & 11.7 & $\ldots$ \\
\hline $6 \ldots \ldots \ldots \ldots$ & 5816.4 & 2.4812 & -5193 & $4.54 \pm 0.45$ & $>0.373$ & $>0.466$ & 11.4 & $\ldots$ \\
\hline $7 \ldots \ldots \ldots \ldots$ & 5816.6 & 2.4813 & -5181 & $7.15 \pm 1.34$ & $>0.225$ & $>0.255$ & 11.4 & $\ldots$ \\
\hline \multicolumn{9}{|l|}{ Si II $\lambda 1526:$} \\
\hline 1 . .................... & 5306.3 & 2.4757 & -5670 & $11.46 \pm 0.35$ & $>0.727$ & $>1.298$ & 13.5 & $\ldots$ \\
\hline $2 \ldots \ldots \ldots \ldots$ & 5307.5 & 2.4764 & -5606 & $12.22 \pm 1.40$ & $>0.296$ & $>0.351$ & 13.0 & $\ldots$ \\
\hline $3 \ldots \ldots \ldots \ldots$ & 5310.1 & 2.4781 & -5459 & $11.23 \pm 0.51$ & $>0.322$ & $>0.388$ & 13.0 & $\ldots$ \\
\hline $4^{\mathrm{g}} \ldots \ldots \ldots \ldots$ & 5310.7 & 2.4785 & -5422 & $10.73 \pm 0.14$ & 1.000 & & $14.1^{\mathrm{e}}$ & $\ldots$ \\
\hline $5 \ldots \ldots \ldots \ldots$ & 5312.5 & 2.4797 & -5322 & $8.15 \pm 0.37$ & $>0.398$ & $>0.507$ & 12.9 & $\ldots$ \\
\hline $6 \ldots \ldots \ldots \ldots$ & 5313.7 & 2.4805 & -5252 & $4.93 \pm 0.11$ & $>0.803$ & $>1.625$ & 13.2 & $\ldots$ \\
\hline 7 …............ & 5314.8 & 2.4812 & -5193 & $5.63 \pm 0.31$ & $>0.497$ & $>0.687$ & 12.9 & $\ldots$ \\
\hline $8 \ldots \ldots \ldots \ldots$ & 5315.1 & 2.4814 & -5174 & $13.30 \pm 0.55$ & $>0.464$ & $>0.623$ & 13.2 & $\ldots$ \\
\hline \multicolumn{9}{|l|}{$\mathrm{Fe}$ II $\lambda 1608:$} \\
\hline $1 \ldots \ldots \ldots \ldots$ & 5595.1 & 2.4786 & -5422 & $5.59 \pm 0.27$ & $>0.580$ & $>0.868$ & 13.3 & $\cdots$ \\
\hline \multicolumn{9}{|c|}{ System C, $z_{\mathrm{abs}}=2.54$} \\
\hline \multicolumn{9}{|l|}{ C IV $\lambda 1548:$} \\
\hline $1 \ldots \ldots \ldots \ldots$ & 5475.9 & 2.5369 & -431 & $11.11 \pm 0.19$ & 1.000 & 0.860 & 13.1 & $\ldots$ \\
\hline \multicolumn{9}{|c|}{ System $\mathrm{D}, z_{\mathrm{abs}}=2.55$} \\
\hline \multicolumn{9}{|l|}{ C IV $\lambda 1548:$} \\
\hline 1 ..................... & 5500.2 & 2.5526 & +896 & $9.40 \pm 0.51$ & 0.787 & 0.864 & 12.9 & 13.0 \\
\hline $2 \ldots \ldots \ldots \ldots$ & 5500.5 & 2.5528 & +914 & $7.64 \pm 1.49$ & 1.000 & 0.297 & 12.5 & \\
\hline $3 \ldots \ldots \ldots \ldots$ & 5500.9 & 2.5531 & +939 & $12.48 \pm 0.30$ & 0.991 & 6.813 & 13.9 & 14.0 \\
\hline $4^{\mathrm{d}} \ldots \ldots \ldots \ldots$ & 5501.5 & 2.5535 & +970 & $10.14 \pm 0.15$ & 1.000 & $\ldots$ & $14.6^{\mathrm{e}}$ & $\cdots$ \\
\hline
\end{tabular}

a Optical depth: For the $\mathrm{C}$ Iv line, it is evaluated for the bluer component in the case $C_{f}$ is the value of col. (6). For a single line, it is evaluated in the case $C_{f}=1.0$.

${ }^{\mathrm{b}}$ Error of $1 \sigma$ is evaluated with $10 \mathrm{~km} \mathrm{~s}^{-1}$ velocity width, because the line profile is very broad and the line center is uncertain.

${ }^{\mathrm{c}}$ It is evaluated for blue component.

${ }^{\mathrm{d}}$ Center of the blue component reaches almost zero flux.

e It is evaluated by fitting the Voigt profile directly.

${ }^{f}$ This line is blended with the blue wing of the C IV line at $z_{\mathrm{abs}}=2.4805$.

$\mathrm{g}$ Center of line reaches almost zero flux. 


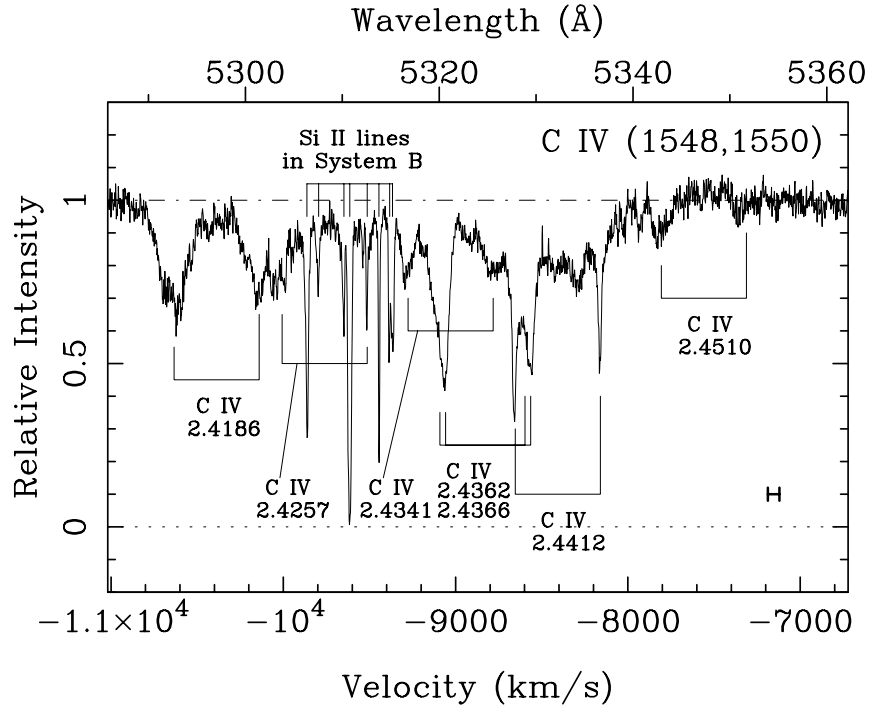

FIG. 4.- C IV components of system A. The horizontal axis denotes the velocity shift from the quasar and the observed wavelength. The narrow lines at $-9800 \mathrm{~km} \mathrm{~s}^{-1}<\Delta v<-9400 \mathrm{~km} \mathrm{~s}^{-1}$ are the Si II 1526 lines of system B. The thick line at lower right denotes 10 times the width of the instrumental profile $\left(66.7 \mathrm{~km} \mathrm{~s}^{-1}\right)$.

$z_{\mathrm{abs}}=2.4367$ detected in D99 has disappeared in our spectrum. But this line could be just a glitch because it looks narrower than the resolution of the spectrum in D99.

System $B\left(z_{\mathrm{abs}}=2.48\right)$. This is the strongest C IV line in the MMT spectrum (D99). Our spectrum resolves this line into 18 narrow components with relatively small Doppler parameters of $4 \mathrm{~km} \mathrm{~s}^{-1} \leq b \leq 25 \mathrm{~km} \mathrm{~s}^{-1}$ (Fig. 5), and most of them can be explained by thermal broadening. Relatively low ionization lines such as Si II $\lambda 1526, \mathrm{Al}$ II $\lambda 1670$, and Fe II $\lambda 1608$ associated with the $\mathrm{C}$ Iv lines are also detected (Fig. 5 ). The strongest components of the low-ionization lines are observed at the center of this system, while the weak components are almost symmetrically arranged at each side of the strongest one.

System $C\left(z_{\mathrm{abs}}=2.54\right)$. This is one of the two systems within $1000 \mathrm{~km} \mathrm{~s}^{-1}$ from the quasar. The line itself is narrow and not resolved into multiple components (Fig. 6). There are no other significant metal lines at this redshift.

System $D\left(z_{\mathrm{abs}}=2.55\right)$. This is another system within $1000 \mathrm{~km} \mathrm{~s}^{-1}$ from the quasar, though this system is redshifted from the quasar. This system is resolved into four narrow components (Fig. 7). The central two components reach almost zero intensity at the bottom. These systems also do not show up in any other metal lines.

\section{CLASSIFICATION OF C IV LINES}

We detected as many as $30 \mathrm{C}$ IV, seven $\mathrm{Al}$ II, eight Si II, and one $\mathrm{Fe}$ II line with velocity differences in the range $-10,600 \mathrm{~km} \mathrm{~s}^{-1}<\Delta v<+1000 \mathrm{~km} \mathrm{~s}^{-1}$ from the quasar. While all the metal lines discovered are relatively close to the quasar in terms of the velocity difference, they could be produced by gas clouds that are either intrinsically associated with the quasar or in intervening galaxies. With the HDS spectrum, we examine the line profiles in detail to classify them as QIALs and SIALs. Among the seven criteria to distinguish QIALs from SIALs described in $\S 1$, we mainly

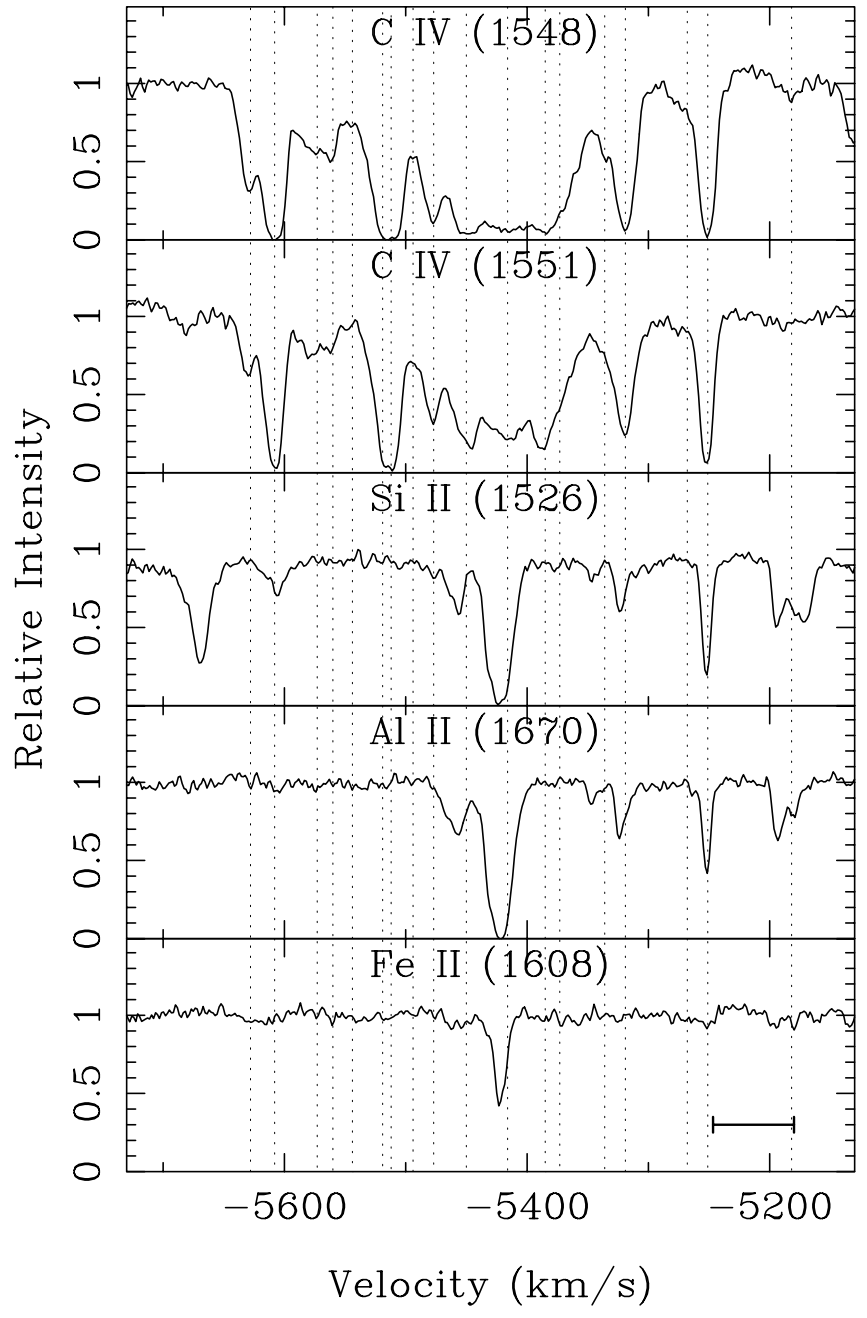

FIG. 5.- C IV components of system B. The horizontal axis denotes the velocity shift from the quasar. The vertical dotted lines denote the positions of the $\mathrm{C}$ IV 1548 components. The thick line at lower right in the Fe II plot denotes 10 times the width of the instrumental profile $\left(66.7 \mathrm{~km} \mathrm{~s}^{-1}\right)$.

use criteria (1), (3), and (5), which can be directly applied to our spectrum.

\subsection{Absorption-Line Profile}

Absorbers that produce very broad and smooth profiles are expected to be intrinsically associated with the quasar nuclear region. For example, the broad absorption line (BAL) systems with $b \sim 10^{4} \mathrm{~km} \mathrm{~s}^{-1}$ are considered to have originated in gas motion in the broad-line regions of quasars. On the other hand, intervening absorption lines that are inherent in cosmologically intervening galaxies have a narrow profile with $b \leq 12 \mathrm{~km} \mathrm{~s}^{-1}$, which corresponds to gas temperature $T \sim 10^{5} \mathrm{~K}$ for the $\mathrm{C}$ IV ion.

System A has five relatively broad C IV lines $(b \geq 65 \mathrm{~km}$ $\mathrm{s}^{-1}$ ), and all them have relatively smooth line profiles and are not resolved into narrow components even in our highresolution spectrum. Their broad-line profiles with $b \geq 65$ $\mathrm{km} \mathrm{s}^{-1}$, where $b=\left(b_{T}^{2}+b_{\text {tur }}^{2}\right)^{1 / 2}$, are not likely to be produced by only thermal broadening, $b_{T}$, because the corresponding temperature is very high, $T \geq 3 \times 10^{6} \mathrm{~K}$, compared with typical metal line systems. The microturbulence, $b_{\text {tur }}$, is expected to contribute to the majority of the Doppler parameter. Therefore the results strongly suggest 


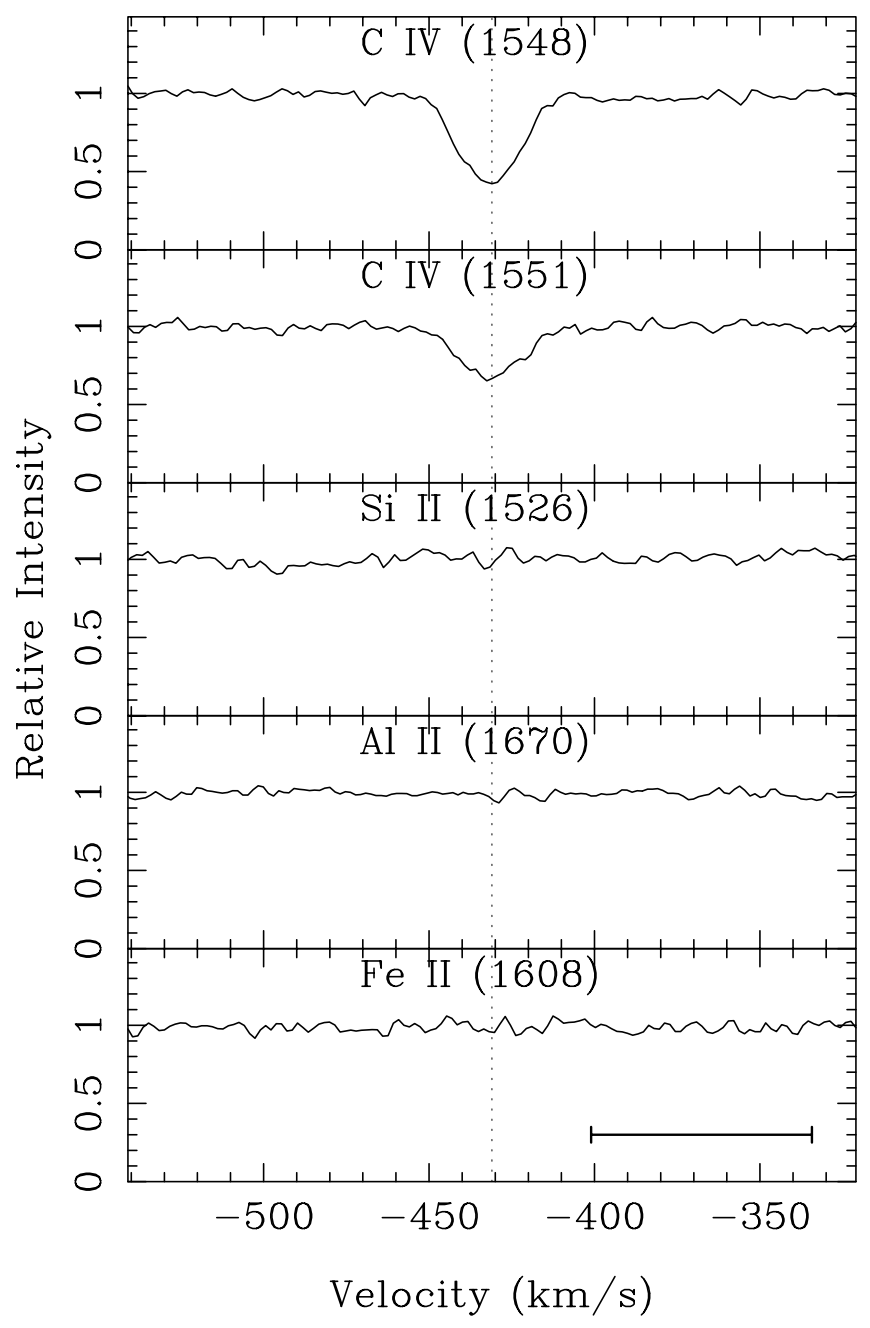

FIG. 6.-Same as Fig. 5, but for system C

that these lines are intrinsic ones, which occur in the vicinity of the quasar nucleus although their Doppler parameters are too small compared with those of BALs.

On the other hand, systems B, C, and D consist of only narrow components with $b \leq 25 \mathrm{~km} \mathrm{~s}^{-1}$ (the corresponding temperature is $T \leq 4.5 \times 10^{5} \mathrm{~K}$ for the $\mathrm{C}$ IV ion), and $65 \%$ of them have $b<12 \mathrm{~km} \mathrm{~s}^{-1}$, statements that imply these systems can be produced by intervening galaxies. The component at $z_{\mathrm{abs}}=2.4412$ in system A also has a relatively narrow line profile.

\subsection{Partial Coverage}

We evaluate the covering factor, $C_{f}$, the line-of-sight coverage fraction of the absorber over the continuum source. Since the components of absorption systems seem to be fully resolved in our spectrum, we can evaluate the covering factors for $\mathrm{C}$ IV doublets by using the following equations:

$$
C_{f}= \begin{cases}\frac{R_{r}^{2}-2 R_{r}+1}{R_{b}-2 R_{r}+1} & \text { for } R_{r}>R_{b} \geq R_{r}^{2}, \\ 1 & \text { for } R_{b}<R_{r}^{2}, \\ 1-R_{r} & \text { for } R_{b} \geq R_{r},\end{cases}
$$

where $R_{b}$ and $R_{r}$ are residual fluxes at the line centers for the

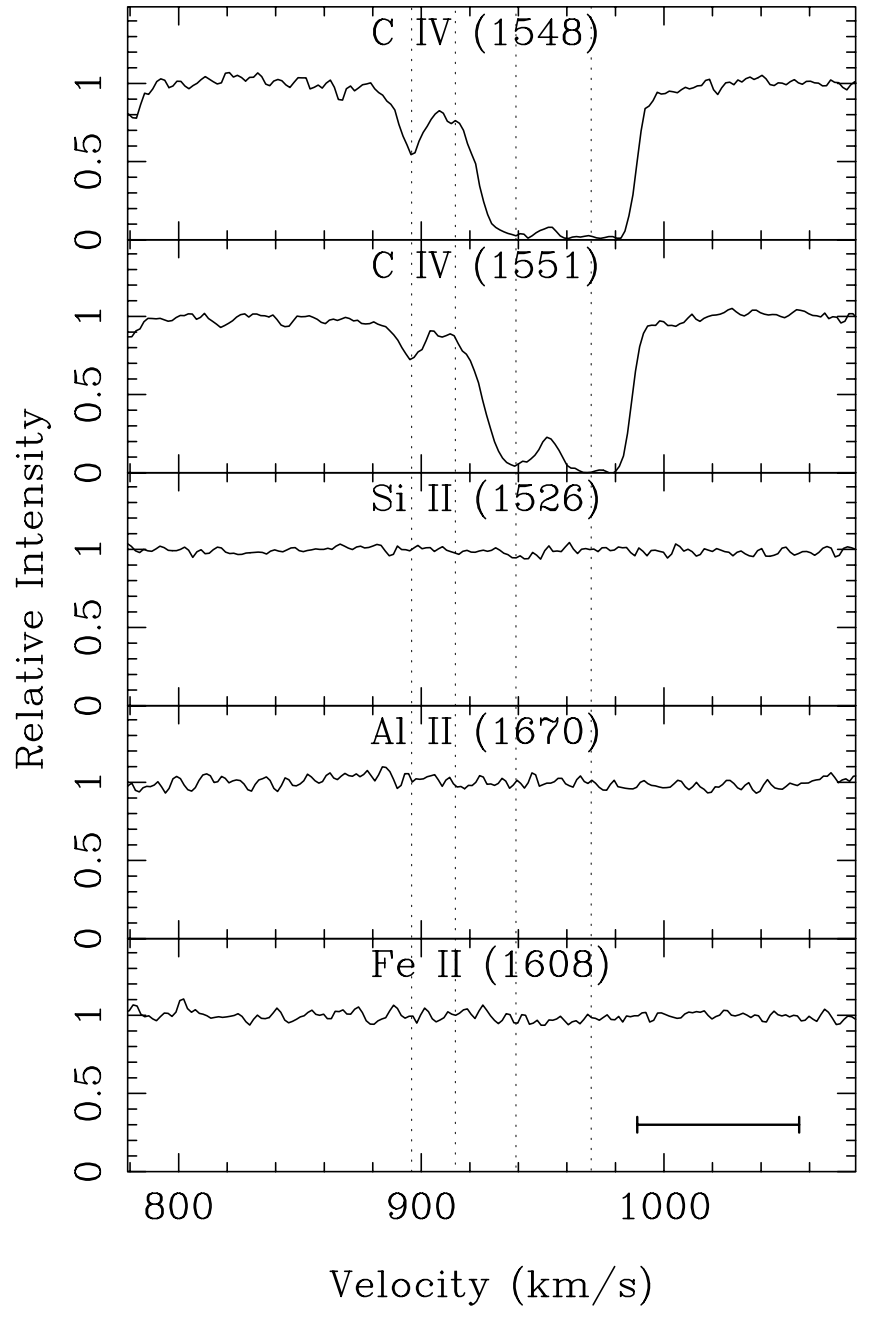

Fig. 7.- Same as Fig. 5, but for system D

blue ( $\mathrm{C}$ IV $\lambda 1548)$ and red ( $\mathrm{C}$ IV $\lambda 1551)$ components of the doublet lines on the normalized spectrum (Hamann et al. 1997b; Barlow \& Sargent 1997).

We also derived the optical depth, $\tau$, at the line center and the total column density, $\log N$, for the blue component by using the following equations:

$$
\begin{gathered}
\tau=-\ln \frac{R_{b}-1+C_{f}}{C_{f}}, \\
\log N=\log \frac{\tau b}{f \lambda_{0}}+14.576,
\end{gathered}
$$

where $f$ and $\lambda_{0}$ are the oscillator strength and the resonance wavelength of the absorption line, respectively (Hamann et al. 1997b; Barlow \& Sargent 1997; Savage \& Sembach 1991; Peterson 1997). For some C IV doublets, the blue components reach almost zero flux, and the residual flux is very uncertain. In that case, we assume that $C_{f}=1$ and evaluate column densities by directly fitting the Voigt profile for them. The $C_{f}, \tau$, and $\log N$ values of $30 \mathrm{C}$ IV components are listed in Table 1. For C Iv lines with $C_{f}<1$, we also evaluate $\log N$ in the case of $C_{f}=1$ for comparison.

For single lines such as $\mathrm{Al}$ II, Si II, and Fe II, we evaluated only the lower limits for $C_{f}$, and we evaluated $C_{f}$ and $\tau$ by 
using

$$
\begin{gathered}
C_{f} \geq 1-R_{0}, \\
\tau \geq-\ln R_{0} .
\end{gathered}
$$

We also evaluated $\log N$ in the case of $C_{f}=1$ by using equation (4).

The Al II $\left(z_{\mathrm{abs}}=2.4785\right)$ and Si II $\left(z_{\mathrm{abs}}=2.4785\right)$ components in system B reach almost zero flux at the line centers. We assume $C_{f}=1$ for them and evaluated column densities by directly fitting the Voigt profile.

For system A, we evaluated these parameters for only two C IV lines $\left(z_{\mathrm{abs}}=2.4186\right.$ and 2.4412), because other lines are heavily blended with each other or with the Si II lines of system B. For the C IV line at $z_{\mathrm{abs}}=2.4186$, we also evaluated $1 \sigma$ errors of the parameters measured at the bottom of the line with $10 \mathrm{~km} \mathrm{~s}^{-1}$ velocity width, because this line has a very broad line profile (FWHM $>150 \mathrm{~km} \mathrm{~s}^{-1}$ ) and the line center is uncertain.

The $\mathrm{C}$ IV line $\left(z_{\mathrm{abs}}=2.4186\right)$ in system $\mathrm{A}$ is likely to be a QIAL, because the line has $C_{f}=0.31$. On the other hand, the C IV line at $z_{\mathrm{abs}}=2.4412$ has $C_{f}=0.8$, although the value is uncertain because of its blending with broad $\mathrm{C}$ IV lines at the same wavelength region. If those broad $\mathrm{C}$ IV lines are removed properly from the spectrum, the $C_{f}$ of the C IV line tends to increase toward 1.

The result of the covering factor analysis suggests that systems B, C and D cover the quasar completely. The mean $C_{f}$ values of 18,1 , and $4 \mathrm{C}$ iv components in systems B, C and $\mathrm{D}$ are almost unity, $0.943,1.00$ and 0.945 , respectively, and $56 \%, 100 \%$, and $50 \%$ of C IV components in these systems have $C_{f}=1$. Only the C IV line at $z_{\text {abs }}=2.4803$ in system B has an exceptionally small covering factor, $C_{f}=0.277$. But this value is tentative, because this line is heavily blended with the blue wing of the $\mathrm{C}$ IV line at $z_{\text {abs }}=2.4805$.

\subsection{Time Variability}

Time variability can be a powerful tool in identifying QIALs, simply because once the variability is confirmed, the line is most likely to be a QIAL (but QIALs are not always time variable). There were examples that show this method is indeed very valuable. Absorption lines with time variability were found at $z_{\mathrm{abs}}=2.13$ on the spectrum of UM 675 (Hamann et al. 1995), and at $z_{\mathrm{abs}}=2.24$ on the spectrum of Q2343+125 (Hamann et al. 1997a). Equivalent widths of these lines varied by factors of 3 and 4 within 12 and $0.3 \mathrm{yr}$, respectively.

The Fe II $\lambda 1608$ line at $z_{\mathrm{abs}}=2.4367$ in system A shows large time variability, though the identification of this line by D99 probably was not very secure. This line had disappeared in our spectrum, although it had been strong $\left(W_{\mathrm{rf}}=0.11 \AA\right.$ ) in D99 (it is seen around $5525 \AA$ in Fig. 3, bottom). The quantitative comparison of the equivalent width of the line in our spectrum with that of D99 is not given, because the spectral resolution was not identical. Nonetheless, if the line identified by D99 is confirmed as a real $\mathrm{Fe}$ II line at $z_{\mathrm{abs}}=2.4367$, the presence of the time variability is evident and this line is probably associated with the quasar.

\section{RESULTS AND DISCUSSION}

Here we classify the detected C IV lines into QIALs and SIALs, based on the results in the previous section. After that, we consider the origin(s) of the absorption lines and the cause of the large number density of $\mathrm{C}$ IV lines in the spectrum of high-redshift luminous quasar HS $1603+3820$.

\subsection{Result of Classification Based on Three Criteria 5.1.1. System A}

In system A, all five broad C IV lines have similar smooth line profiles, and they are detected within $3000 \mathrm{~km} \mathrm{~s}^{-1}$ from each other. One of them, at $z_{\mathrm{abs}}=2.4186$, which corresponds to $\Delta v=-10,600 \mathrm{~km} \mathrm{~s}^{-1}$ from HS $1603+3820$, is confirmed to have a small covering factor of $C_{f} \sim 0.31$. For this $\mathrm{C}$ IV line, the FWHM, column density, and covering factor can be properly measured; they are $190 \mathrm{~km} \mathrm{~s}^{-1}, 15.8$, and 0.31 , respectively, and are in a similar range of values to those of the already confirmed QIALs, $440 \mathrm{~km} \mathrm{~s}^{-1}, 15.2$, and 0.19 for a C Iv line at $-24,000 \mathrm{~km} \mathrm{~s}^{-1}$ from Q2343+125 (Hamann et al. 1997a), and $56 \mathrm{~km} \mathrm{~s}^{-1}, 14.1$, and 0.31 for a C Iv line at $-1500 \mathrm{~km} \mathrm{~s}^{-1}$ from UM 675 (Hamann et al. 1997b). The other broad lines in system A cannot be fitted individually because of line blending. Nonetheless, they are very likely to be QIALs, not only because they have lower ejection velocities (nearer to the quasar) than the $\mathrm{C}$ Iv line at the lowest redshift $\left(z_{\text {abs }}=2.4186\right)$ but also because they have similar line profiles to the fitted C IV line.

On the other hand, there is at least one narrow $\mathrm{C}$ IV line with $b \sim 16 \mathrm{~km} \mathrm{~s}^{-1}\left(z_{\mathrm{abs}}=2.4412\right)$ in the system, and this line is confirmed to have a large covering factor, $C_{f}=0.8$, with the uncertainty of merging with other broad $C_{\text {IV }}$ lines. The covering factor of the line is large: $C_{f}$ is close to unity, which also supports the idea that the line is a SIAL. If this is true, the association of the narrow line and the broad lines in system $\mathrm{A}$ is just a chance projection.

There are at least two possible origins for absorbers that produce QIALs in system A. First, we consider the possibility that they are in fact similar absorbers that produce much broader lines in the spectra of the so-called broad absorption line (BAL) quasars. BAL absorbers, which produce lines with $b=10,000-20,000 \mathrm{~km} \mathrm{~s}^{-1}$, are thought to be at a distance of a few tens of parsecs from the continuum source of quasars (see Weymann, Turnshek, \& Christiansen 1985; Turnshek et al. 1985). Although widths of the C Iv lines in system A with $b=60-120 \mathrm{~km} \mathrm{~s}^{-1}$ are much smaller than typical BALs, they can be seen in the spectrum of the quasar at a phase in its transition from (to) a quasi-BAL to (from) a standard quasar (Morris et al. 1986; Richards et al. 2002a). When a BAL is formed many narrow components could blend with each other and make broad absorption features (BAL) as time passes, or when a BAL dies most of the optically thin gas of BAL absorbers dissipates and only dense cores that produce narrow lines are left. Ganguly, Charlton, $\&$ Bond (2001) also offered the sporadic (or quasi-periodic) mass ejection scenario, which would have a density structure of the outflowing wind and produce narrow absorption line clustering.

Another candidate is outflowing clouds accelerated with the jets from the central engine of the quasar. In this case, the absorbing gas extends to distances of hundreds of kiloparsecs (Bridle \& Perley 1984). According to the small covering factor of the $\mathrm{C}$ IV line at $z_{\mathrm{abs}}=2.4186$, however, this interpretation is not favorable because it is difficult for the 
absorbers at a distance of hundreds of kiloparsecs from the quasar to cover the continuum source partially.

Based on these points, the broad C IV lines in system A are probably produced by outflowing absorbers very close to the continuum source. On the other hand, the narrow $\mathrm{C}$ IV line at $z_{\mathrm{abs}}=2.4412$ could be a SIAL that arises in an intervening absorber or a QIAL produced by gas cloud outflowing with almost the same velocity as absorbers of the broad lines, though they are at high altitude because its covering factor is close to unity.

Another remarkable point is that the $\mathrm{Fe}$ II line at $z_{\text {abs }}=2.4367$ in D99 shows time variability, which suggests the line is a QIAL. However, the relationship between this line and the corresponding C IV line at nearly the same redshift is unclear. Identification of the line as Fe II $\lambda 1608$ in D99 seems to be based on the detection of $\mathrm{C}$ IV lines at the same redshift. In our spectrum, in which the line is not seen, however, we still have the corresponding $\mathrm{C}$ Iv line at nearly the same redshift $\left(z_{\mathrm{abs}}=2.4366\right)$. In fact, the line has a relatively narrow line width, $b=25.3 \mathrm{~km} \mathrm{~s}^{-1}$, which can be interpreted as a SIAL. If this C Iv line is really a SIAL, we argue that the identification of the line at $5525 \AA$ as Fe II $\lambda 1608$ in D99 may be reconsidered while there are other possibilities that (1) there was another C IV system at the same redshift that disappeared with $\mathrm{Fe}$ II or (2) the $\mathrm{C}$ IV line is a QIAL but only the Fe II line has disappeared.

\subsubsection{System B}

All the C IV components in system B have narrow-line profiles, and they have large covering factors. System B was plausibly produced by clumpy gas clouds in an intervening galaxy. The narrow components distribute within $\pm 250 \mathrm{~km}$ $\mathrm{s}^{-1}$, which can be explained by the bulk motions in the galaxy. The column density distributions of the metal lines $\mathrm{C}$ IV, $\mathrm{Al}$ II, Si II, and Fe II are plotted in Figure 8. The highvalence ion (C IV) distributes more or less evenly within $\pm 250 \mathrm{~km} \mathrm{~s}^{-1}$ from the center of system B. On the other hand, lines of low-valence ions (Al II, Si II, and Fe II) with large column density are seen only near the velocity center; this core-halo structure of low- and high-ionization lines also supports the idea that the lines have arisen in a galaxy. Gas density in halo clouds is so small that they are easily affected and highly ionized by background UV flux.

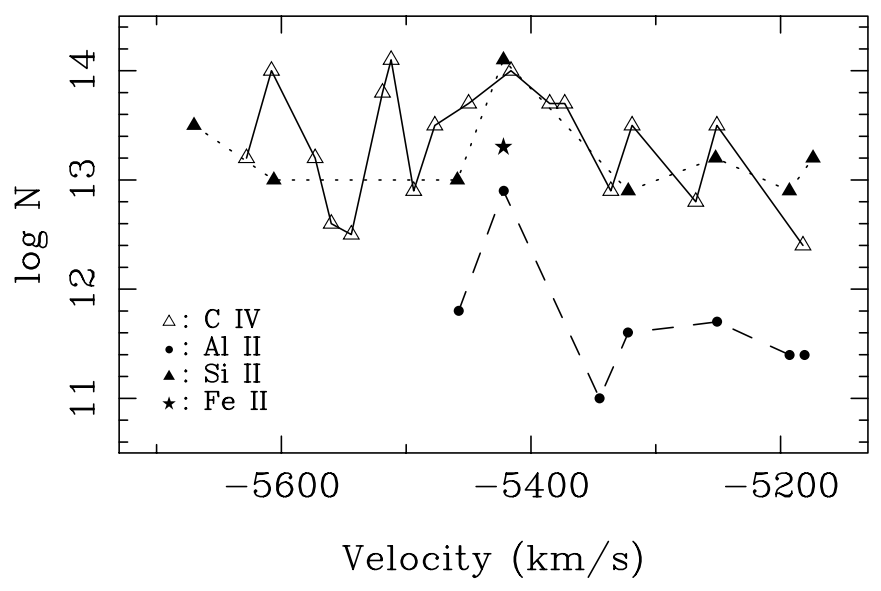

FIG. 8.-Distributions of column density for the $\mathrm{C}$ IV, Si II, Al II, and Fe II lines in system B, showing the C IV (open triangles), Si II (filled triangles), $\mathrm{Al}$ II (circles), and Fe II (star) lines.

\subsubsection{Systems $C$ and $D$}

Systems C and D are detected within $1000 \mathrm{~km} \mathrm{~s}^{-1}$ from the quasar redshift. On one hand, the covering factors of the lines in these systems are near unity, which suggests that they are separated from the continuum source far enough to cover the quasar entirely in the direction of line of sight. On the other hand, their small velocity differences of $430 \mathrm{~km} \mathrm{~s}^{-1}$ blueward (system C) and $950 \mathrm{~km} \mathrm{~s}^{-1}$ redward (system D) from the quasar redshift imply that they could be physically associated with the quasar.

There are at least two interpretations for these systems. First, the lines may be produced by the intervening galaxies in the vicinity of the quasar, which moves around the quasar-like cluster (group) of galaxies. The velocity differences of the systems from the quasar $\left(\Delta v=430\right.$ and $\left.950 \mathrm{~km} \mathrm{~s}^{-1}\right)$ are within the typical velocity dispersion in rich clusters ( $\sigma \sim 1000 \mathrm{~km} \mathrm{~s}^{-1}$, for the richest nearby cluster of galaxies). The large covering factors, $C_{f} \sim 1$, of these systems can be easily produced by such intervening galaxies. Second, the lines could be produced by gas clouds intrinsically associated with the quasar, because systems $\mathrm{C}$ and $\mathrm{D}$ do not have low-ionization lines such as $\mathrm{Si}$ II, $\mathrm{Al}$ II, and Fe II, which suggests they are on a high ionization level. The strong UV flux from the quasar can make them highly ionized. In the diskwind model (Murray \& Chiang 1995, 1998) that has been used to explain BAL quasars, intrinsic gas clouds exist along the windstream lines from the continuum source to a relatively large distance. The simulations by Proga, Stone, \& Kallman (2000) show that once outflowing gas clouds reach a high altitude, they return toward the center along the outflowing stream, which can produce not only blueshifted but also redshifted absorption lines relative to the systemic redshift of the quasar simultaneously. Therefore system $\mathrm{C}$ is outflowing from the quasar, while system D has already reached a high altitude and flows into the central source of the quasar. These systems should be separated far enough from the quasar to cover the quasar entirely.

\subsection{Origin of $C$ IV Line Clustering}

If systems $\mathrm{C}$ and $\mathrm{D}$ have arisen in the galaxies around the quasar, that is, if they are SIALs, then five of eight $\mathrm{C}$ IV lines detected in D99 at $2.42<z_{\text {abs }}<2.55$ are QIALs and three are SIALs. The number density of SIALs per unit redshift is $N(z)=8.82$ at $N(z)=2.38$. On the other hand, if systems C and $\mathrm{D}$ are classified as QIALs, the corresponding number density is $N(z)=2.94$, which is almost consistent with the expected value, $N(z)=2.45_{-0.49}^{+0.60}$, at $N(z)=2.40$ (Misawa et al. 2002). Therefore the number density excess of C IV absorption lines at $N(z)=z_{\mathrm{em}}$ in the spectrum of $\mathrm{HS}$ $1603+3820$ may be caused by the number density excess of QIALs.

Foltz et al. (1986) found that the number density of C IV absorption lines increases near the emission redshift of the radio-loud quasars with their $\sim 1 \AA$ resolution spectra. We evaluated the number densities of $\mathrm{C}$ IV absorption lines for the five quasars in the sample of Foltz et al. (Q1256+357, Q1416+067, Q1445+335, Q1634+176, and Q1756+237) that have strong absorption complexes near the quasars. We used the range of spectra between $z_{15}$ (redshift 15,000 $\mathrm{km} \mathrm{s}^{-1}$ blueward from $z_{\mathrm{em}}$ ) and $z_{\mathrm{em}}$ or $z_{\text {high }}$ (the redshift at which the $\mathrm{C}$ IV absorption line of highest $z$ is detected beyond $z_{\mathrm{em}}$ ) over which the $4 \sigma$ detection limit corresponds to $W_{\mathrm{rf}}=0.2 \AA$. The mean value of the C Iv number density 
(not a Poisson sample) is $\langle N(z)\rangle=36.2 \pm 10.0$ per redshift at $z_{\text {abs }} \sim 1.65$. With the $\sim 1.5 \mathrm{~A}$ resolution spectrum of $\mathrm{D} 99$, the number density of the C IV line for HS $1603+3820$ is evaluated to be 43.5 between $z_{15}$ and $z_{\text {high }}\left(z_{\text {abs }}\right.$ of system D) over which the $4 \sigma$ detection limit is $W_{\mathrm{rf}}=0.15 \AA$. The $N(z)$ for HS $1603+3820$ has a large value about the same as the mean value $N(z)$ for six quasars in Foltz et al. (1986), though the detection limit of equivalent width and spectrum resolution are slightly different. These number density excesses of $\mathrm{C}$ IV lines at $z_{\mathrm{abs}} \sim z_{\mathrm{em}}$ could be produced by QIALs.

\section{CONCLUSIONS AND FUTURE WORK}

We have analyzed the $\mathrm{C}$ IV absorption-line clustering at $z_{\mathrm{abs}} \sim z_{\mathrm{em}}$ in the spectrum of HS $1603+3820$ by using Subaru and HDS data. By using three properties of $\mathrm{C}$ IV lines, specifically time variability, covering factor, and absorption-line profile, we have confirmed that among four C IV systems one system is almost certainly intrinsic to the quasar and one system has probably arisen in an intervening galaxy. The other two systems, at $z_{\text {abs }} \sim z_{\text {em }}$, have properties that are consistent with either interpretation. If they are classified as QIALs, the number density of SIALs in the Poisson sample becomes almost consistent with the expected value at the similar redshift far from quasars. In Figure 9, we summarize the distributions of four C IV systems in the physical distance from the quasar in the cases in which systems C and D are classified as SIALs and QIALs.

We finally note some future prospects. In the course of this study, we have not applied the time variability analysis to systems B, C, and D, because the resolution of our spectrum is different from that of D99. An additional spectrum taken with the same configuration as our spectrum a few months to years later might enable us to confirm the time variability of these systems. Deep imaging observation with narrowband filters for detecting $\mathrm{Ly} \alpha$ or $\mathrm{H} \alpha$ emission lines of galaxies around the quasar will be also useful to under-
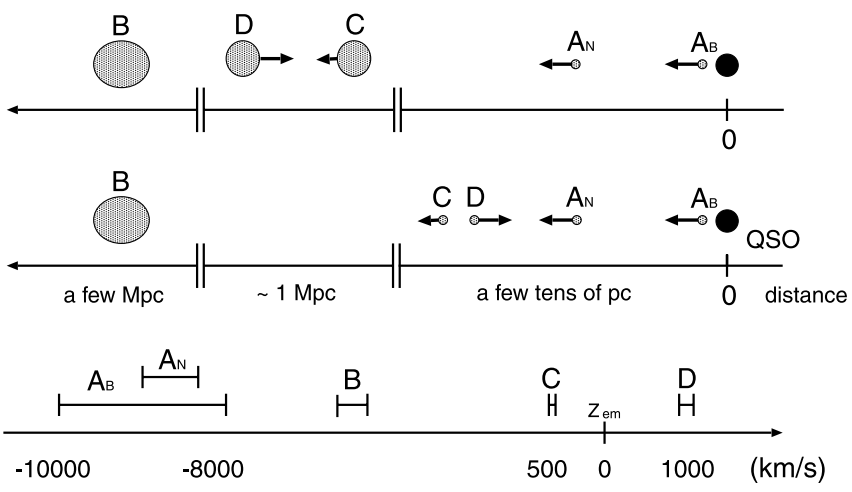

FIG. 9.-Cartoons of the structure of absorbers at $z_{\mathrm{abs}} \sim z_{\mathrm{em}}$ in the line of sight to the quasar HS $1603+3820$. Top and middle: Distribution of four systems in physical distance from the quasar if systems C and D are classified as SIALs and QIALs. Positions of systems C and D are interchangeable. Bottom: Distribution of the systems in the observed velocity shift from the quasar.

stand the true origins of systems $\mathrm{C}$ and $\mathrm{D}$. If many galaxies are detected around the quasar at the redshift around $z_{\mathrm{em}}$ of the quasar, they would support the idea that systems $\mathrm{C}$ and $\mathrm{D}$ are produced by intervening galaxies in the neighborhood of the quasar.

We are grateful to the staff of the Subaru Telescope, which is operated by the National Astronomical Observatory of Japan. We would like to thank K. Kawabata, S. Kawanomoto, W. Aoki, and N. Suzuki for their advice about data reduction. We also thank R. F. Carswell, J. K. Webb, A. J. Cooke, and M. J. Irwin for their VPFIT software package, available in their web site. We wish to thank the anonymous referee for the report, which improved the clarity of this presentation.

\section{REFERENCES}

Barlow, T. A., Junkkarinen, V. T., Burbidge, E. M., Weymann, R. J., Morris, S. L., \& Korista, K. T. 1992, ApJ, 397, 81

Barlow, T. A., \& Sargent, W. L. W. 1997, AJ, 113, 136

Bridle, A. H., \& Perley, R. A. 1984, ARA\&A, 22, 319

Carswell, R. F., Webb, J. K., Baldwin, J. A., \& Atwood, B. 1987, ApJ, 319, 709

Dobrzycki, A., Engels, D., \& Hagen, H.-J. 1999, A\&A, 349, L29 (D99)

Dobrzycki, A., Engels, D., Hagen, H.-J., Elvis, M., Huchra, J., \& Reimers, D. 1996, BAAS, 188(0602)

Espey, B. R. 1993, ApJ, 411, L59

Foltz, C. B., Weymann, R. J., Peterson, B. M., Sun, L., Malkan, M. A., \& Chaffee, F. H., Jr. 1986, ApJ, 307, 504

Ganguly, R., Charlton, J. C., \& Bond, N. A. 2001, ApJ, 553, L101

Goodrich, R. W., \& Miller, J. S. 1995, ApJ, 448, L73

Hagen, H.-J., Groote, D., Engels, D., \& Reimers, D. 1995, A\&AS, 111, 195

Hamann, F., Barlow, T. A., Beaver, E. A., Burbidge, E. M., Cohen, R. D., Junkkarinen, V., \& Lyons, R. 1995, ApJ, 443, 606

Hamann, F., Barlow, T. A., \& Junkkarinen, V. 1997a, ApJ, 478, 87

Hamann, F., Barlow, T. A., Junkkarinen, V., \& Burbidge, E. M. 1997b, ApJ, 478, 80

Misawa, T., Tytler, D., Iye, M., Storrie-Lombardi, L. J., Suzuki, N., \&

Wolfe, A. M. 2002, AJ, 123, 1847

Morris, S. L., Weymann, R. J., Foltz, C. B., Turnshek, D. A., Shectman, S., Price, C., \& Boroson, T. A. 1986, ApJ, 310, 40

Murray, N., \& Chiang, J. 1995, ApJ, 454, L105

. 1998, ApJ, 494, 125

Noguchi, K., et al. 2002, PASJ, 54, in press

Peterson, B. M. 1997, An Introduction to Active Galactic Nuclei (Cambridge: Cambridge University Press)

Proga, D., Stone, J. M., \& Kallman, T. R. 2000, ApJ, 543, 686

Richards, G. T., Gregg, M. D., Becker, R. H., \& White, R. L. 2002a, ApJ, 567, L13

Richards, G. T., Vanden Berk, D. E., Reichard, T. A., Hall, P. B., Schneider, D. P., SubbaRao, M., Thakar, A. R., \& York, D. G. 2002b, AJ, 124, 1

Sargent, W. L. W., Boksenberg, A., \& Steidel, S. S. 1988, ApJS, 68, 539

Savage, B. D., \& Sembach, K. R. 1991, ApJ, 379, 245

Steidel, S. S. 1990, ApJS, 72, 1

Turnshek, D. A., Foltz, C. B., Weymann, R. J., Lupie, O. L., McMahon, R. G., \& Peterson, B. M. 1985, ApJ, 294, L1

Vanden Berk, D. E., et al. 2001, AJ, 122, 549

Webb, J. K. 1987, Ph.D. thesis, Univ. Cambridge

Weymann, R. J., Turnshek, D. A., \& Christiansen, W. A. 1985, in Astrophysics of Active Galaxies and Quasi-Stellar Objects, ed. J. S. Miller (Mill Valley: University Science Books) 